\title{
EPIDEMIOLOGía DEL CARCINOMA PAPILAR DE TIROIDES EN PACIENTES DEL INSTITUTO ONCOLÓGICO JUAN TANCA MARENGO, SOLCA-GUAYAQULL, AÑO 2014
}

\author{
EPIDEMIOLOGY OF PAPILLARY THYROID CARCINOMA IN \\ JUAN TANCA MARENGO CANCER INSTITUTE PATIENTS \\ SOLCA-GUAYAQUIL, IN 2014
}

\author{
EPIDEMIOLOGIA DO CARCINOMA PAPILAR DA \\ TIREÓIDE EM PACIENTES DO INSTITUTO ONCOLÓGICO \\ JUAN TANCA MARENGO, SOLCA-GUAYAQUIL, ANO 2014
}

\begin{abstract}
VALERIA VIVIANA MOREJON COBA, ANDRÉ DAVID ORELLANA JAIME
Universidad Católica de Santiago de Guayaquil; Guayaquil, Ecuador
\end{abstract}

Resumen

Objetivo: determinar la presentación del carcinoma papilar de tiroides en la población atendida en el Instituto Oncológico Juan Tanca Marengo, SOLCA - Guayaquil, en el año 2014. Metodología: estudio epidemiológico, descriptivo, retrospectivo, en una población de 127 pacientes de SOLCA - Guayaquil, diagnosticados con carcinoma papilar de tiroides, año 2014. Resultados: se registra una prevalencia en sexo femenino, con edad promedio de 37 a 52 años. El $60.66 \%$ de la población perteneció a la zona urbana. Mayor frecuencia de afectación del lóbulo derecho de la glándula tiroides. El grupo de edad de 37 a 55 años coincide con la presencia de metástasis de tejidos blandos, ganglionar y combinada. Conclusión: el carcinoma papilar de tiroides se presenta con mayor frecuencia en mujeres con una edad media de 46 años, de zonas urbanas.

PALABRAS CLAVE: Carcinoma papilar, tiroides, epidemiología, población.

Abstract

Objective: determine papillary thyroid carcinoma presentation in the population attended at the Juan Tanca Marengo Cancer Institute, SOLCA - Guayaquil, in 2014. Metodology: epidemiological, descriptive, analytical, inferential, comparative, retrospective study, in a population of 127 patients from SOLCA - Guayaquil. Reference was taken to all patients diagnosed with papillary thyroid carcinoma in 2014. Results: it was registered a prevalence in women, with an average age of 37 to 52 years. $60.66 \%$ of the population belonged to the urban area. Greater frequency involvement of the right lobe of the thyroid gland. The age group of 37 to 55 years coincides with the presence of combined soft tissue, lymph node metastasis. Conclusion: papillary thyroid carcinoma occurs more frequently in women with a mean age of 46 years, from urban areas.

KEYWORDS: papillary carcinoma, thyroid, epidemiology, population.

Resumo

Objetivo: determinar a apresentação do carcinoma papilífero da tireoide na população atendida no Instituto de Câncer Juan Tanca Marengo, SOLCA - Guayaquil, em 2014. Metodologia: estudo epidemiológico, descritivo, analítico, inferencial, comparativo, retrospectivo, em uma população de 127 pacientes do SOLCA - Guayaquil. Todos os pacientes diagnosticados com carcinoma papilífero da tireoide em 2014 foram tomados como referência. Resultados: é registrada uma prevalência em mulheres, com idade média de 37 a 52 anos. 60,66\% da população pertencia à área urbana. Maior frequência de envolvimento do lobo direito da glândula tireóide. A faixa etária de 37 a 55 anos coincide com a presença de tecidos moles combinados, metástase linfonodal. Conclusão: 0 carcinoma papilifero da tireoide ocorre com maior frequência em mulheres com idade média de 46 anos, de áreas urbanas.

PALAVRAS-CHAVE: carcinoma papilar, tireoide, epidemiologia, população. 
INTRODUCCIÓN

El carcinoma de tiroides es la neoplasia endócrina más común. Se clasifica en carcinoma papilar de tiroides (PTC), carcinoma folicular de tiroides (FTC), carcinoma anaplásico de tiroides (ATC), carcinoma medular de tiroides (MTC). Los carcinomas que tienen un buen pronóstico y son curables cuando se los encuentra en las primeras fases de la enfermedad estan el PTC y el FTC. El ATC es el más agresivo ${ }^{1,2,3,4}$. La incidencia del cáncer de tiroides es de 9/100.000 personas en USA, aumenta con la edad y alcanza una meseta después de los 50 años. Los pacientes jóvenes ( $<20$ años) y maduros (>50 años) tienen peor pronóstico. La incidencia es el doble en mujeres de raza blanca que en mujeres de raza negra (11.2 y 5.8 respectivamente). Es más frecuente en el género femenino que en el masculino, con una tasa de presentación de 11 y 5 casos por 100,000 personas respectivamente, pero el género masculino tiene peor pronóstico ${ }^{1,2,3,4,5,6,7}$.

El carcinoma papilar de tiroides es el tipo de cáncer tiroideo más frecuente y constituye del $70-90 \%$ de carcinomas bien diferenciados de tiroides. Clásicamente se lo ha considerado como de buen pronóstico, ${ }^{8}$. La mayoría de los individuos se encuentran eutiroideos y presentan una masa cervical indolora de crecimiento lento; suele ser de presentación multifocal e invadir localmente la glándula tiroidea y extenderse a través de la cápsula tiroidea invadiendo las estructuras adyacentes del cuello; la mayor parte de los canceres papilares que son identificados en los estadios iniciales (estadio I, II 80\%), tienen un buen pronóstico. La mortalidad es mayor en el estadio IV, pero este grupo representa solo el $1 \%$ de los pacientes $2,3,9-14$.

La ecografía es el examen más empleado para detectar nódulos y quistes mayores de $3 \mathrm{~mm}$. Se han descrito numerosas características que sugieren malignidad en los nódulos como: hipoecogenicidad, presencia de microcalcificaciones, bordes irregulares, halo periférico grueso, irregular o ausente, adenopatías satélites y alto flujo intranodular ${ }^{15}$. La punción y aspiración con aguja fina (PAAF) tiroidea es actualmente el método de elección en la aproximación diagnóstica de la patología tiroidea. El rendimiento es alto especialmente para el diagnóstico de PTC, con un $1 \%$ de falsos positivos y $2 \%$ de falsos negativos ${ }^{2,3,9}$.

El Estudio SEER (Surveillance Epidemiology End Results) determinó la incidencia de cáncer tiroideo a partir de 9 registros obtenidos en distintas áreas de Estados Unidos reportándose una incidencia de 3.6 casos x 100.000 por año en 1973 y de 8.7 x 100.000 por año en 2002. Estos datos muestran que la incidencia de cáncer tiroideo aumentó entre esos años 2.4 veces (95\% I.C. 2.2-2.6) ${ }^{17}$.

Datos estadísticos recolectados en la población de este estudio, refieren que este es el séptimo cáncer de mujer más frecuente en el Ecuador, manifestándose entre las edades de 70-74 años, según los registros obtenidos hasta el 2010, registrándose un promedio de 13.1 casos por año a nivel nacional ${ }^{18}$. El referido registro determinó que el carcinoma papilar es el tipo más frecuente; así mismo por la falta de estadísticas actualizadas en Ecuador, se decidió analizar las principales características de este cáncer con pacientes diagnosticados en el 2014. Es de importancia señalar que no todos los pacientes atendidos en SOLCA pertenecían a la ciudad Guayaquil; es por esta razón que la muestra puede ser utilizada como dato estadístico nacional.

\section{METODOLOGÍA}

Se realizó un estudio de prevalencia, año 2014, de los pacientes con diagnóstico de cáncer papilar de tiroides procedentes de la base de datos del Instituto Oncológico Juan Tanca Marengo, SOLCA - Cuayaquil, con el motivo de obtener una muestra de la población ecuatoriana, caracterizando la forma de presentación de esta enfermedad y comparándola con las características resultantes mencionadas en estudios realizados en otros países. La recolección de los pacientes se llevó a cabo por los autores del estudio, siguiendo las recomendaciones de la Declaración Helsinki. Partiendo de aquellos casos con diagnóstico histopatológico de cáncer de tiroides CIE 10 (C73) que constaban en la base de datos de SOLCAGuayaquil, se descartaron aquellos que poseían diagnóstico de carcinoma medular, folicular, mixto o anaplásico de tiroides, procedencia o residencia extranjera al país de estudio y que no posean el historial clínico completo de acuerdo a las variables necesarias para el estudio epidemiológico que se mencionarán más adelante. A partir de esto, se recolectó una muestra de 127 pacientes la cual nos da un margen de error del $6.29 \%$, teniendo un nivel de confianza del $88.5 \%$.

Las variables empleadas: sexo, edad al momento del diagnóstico de la enfermedad, ocupación, lugar de residencia actual; esta variable se subdivide en provincia y cantón para luego ser catalogada en zona rural o urbana. Cabe recalcar que se toma de referencia como zona urbana 
aquellos cantones que consten con una población mayor a 153.000 habitantes, según estadísticas del censo poblacional del 2010 del Instituto Nacional de estadísticas y censo del Ecuador ${ }^{10}$, INEC. Antecedentes familiares de cáncer, lóbulo tiroideo afectado al momento del diagnóstico, metástasis, variable que fue dividida en: casos que no reportan metástasis; metástasis: ganglionar a tejidos blandos y la ganglionar y de tejidos blandos, según la escala de estadiaje del cáncer $(\mathrm{TNM})^{19}$. Adicional a esto se recolectó datos sobre los resultados de PAAF que constaban en las historias clínicas de los pacientes. Se recalca que no todos los PAAF fueron realizados en la institución de base del presente estudio.

El análisis de los datos se realizó en el software SPSS para Mac, y Microsoft Excel para Windows. Se registraron las características cuantitativas como edad mediante promedio, desviación estándar y las variables cualitativas como sexo, ocupación, residencia, antecedentes patológicos personales y familiares, lóbulo afectado, metástasis y PAAF, mediante frecuencia y porcentajes, tablas 1 y 2.

TABLA 1. CARACTERÍSTICAS EPIDEMIOLÓGICAS DEL CARCINOMA PAPILAR DE TIROIDES EN LA MUESTRA (N=127) DE PACIENTES ATENDIDOS EN EL INSTITUTO ONCOLÓGICO DR. JUAN TANCA MARENGO, SOLCA - GUAYAQUIL, EN EL AÑO 2014.

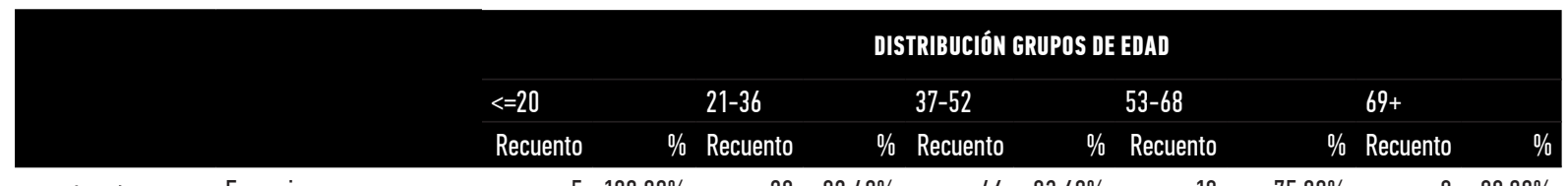

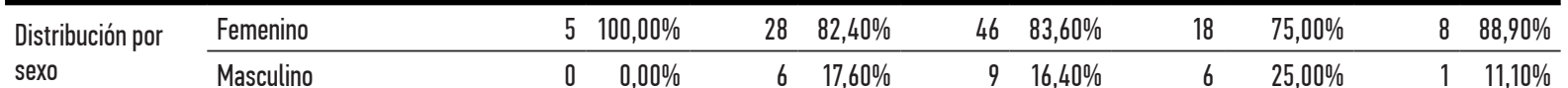

\begin{tabular}{llrrrrrrrrrr} 
sexo & Masculino & 0 & $0,00 \%$ & 6 & $17,60 \%$ & 9 & $16,40 \%$ & 6 & $25,00 \%$ & 1 & $11,10 \%$ \\
\hline \multirow{2}{*}{$\begin{array}{l}\text { Distribución por } \\
\text { zona }\end{array}$} & Urbano & 3 & $60,00 \%$ & 19 & $55,90 \%$ & 35 & $63,60 \%$ & 14 & $58,30 \%$ & 6 & $66,70 \%$ \\
\cline { 2 - 10 } & Rural & 2 & $40,00 \%$ & 15 & $44,10 \%$ & 20 & $36,40 \%$ & 10 & $41,70 \%$ & 3 & $33,30 \%$
\end{tabular}

\begin{tabular}{clllllllllll} 
zona & Rural & 2 & $40,00 \%$ & 15 & $44,10 \%$ & 20 & $36,40 \%$ & 10 & $41,70 \%$ & 3 & $33,30 \%$ \\
\hline & Guayas & 4 & $80,00 \%$ & 16 & $47,10 \%$ & 24 & $43,60 \%$ & 13 & $54,20 \%$ & 6 & $66,70 \%$
\end{tabular}

\begin{tabular}{|c|c|c|c|c|c|c|c|c|c|c|c|}
\hline & Manabí & 1 & $20,00 \%$ & 6 & $17,60 \%$ & 18 & $32,70 \%$ & 5 & $20,80 \%$ & 0 & $33,30 \%$ \\
\hline \multirow{7}{*}{$\begin{array}{l}\text { Distribución por } \\
\text { provincia }\end{array}$} & Los Ríos & 0 & $0,00 \%$ & 2 & $5,90 \%$ & 5 & $9,10 \%$ & 3 & $12,50 \%$ & 1 & $66,70 \%$ \\
\hline & Chimborazo & 0 & $0,00 \%$ & 1 & $2,90 \%$ & 2 & $3,60 \%$ & 0 & $0,00 \%$ & 0 & $0,00 \%$ \\
\hline & Cotopaxi & 0 & $0,00 \%$ & 1 & $2,90 \%$ & 0 & $0,00 \%$ & 0 & $0,00 \%$ & 0 & $11,10 \%$ \\
\hline & Bolívar & 0 & $0,00 \%$ & 1 & $2,90 \%$ & 1 & $1,80 \%$ & 0 & $0,00 \%$ & 0 & $0,00 \%$ \\
\hline & Cañar & 0 & $0,00 \%$ & 0 & $0,00 \%$ & 0 & $0,00 \%$ & 0 & $0,00 \%$ & 1 & $11,10 \%$ \\
\hline & Santa Elena & 0 & $0,00 \%$ & 3 & $8,80 \%$ & 2 & $3,60 \%$ & 0 & $0,00 \%$ & 1 & $0,00 \%$ \\
\hline & El Oro & 0 & $0,00 \%$ & 2 & $5,90 \%$ & 2 & $3,60 \%$ & 3 & $12,50 \%$ & 0 & $0,00 \%$ \\
\hline
\end{tabular}

\begin{tabular}{|c|c|c|c|c|c|c|c|c|c|c|}
\hline El Oro & 0 & $0,00 \%$ & 2 & $5,90 \%$ & 2 & $3,60 \%$ & 3 & $12,50 \%$ & 0 & $0,00 \%$ \\
\hline Esmeraldas & 0 & $0,00 \%$ & 1 & $2,90 \%$ & 0 & $0,00 \%$ & 0 & $0,00 \%$ & 0 & $0,00 \%$ \\
\hline Pichincha & 0 & $0,00 \%$ & 1 & $2,90 \%$ & 1 & $1,80 \%$ & 0 & $0,00 \%$ & 0 & $0,00 \%$ \\
\hline No refiere & 3 & $60,00 \%$ & 27 & $79,40 \%$ & 40 & $72,70 \%$ & 19 & $70,20 \%$ & 4 & $44,40 \%$ \\
\hline
\end{tabular}

Presencia de APF de cáncer

\begin{tabular}{lrrrr} 
No refiere & 3 & $60,00 \%$ & 27 & $79,40 \%$ \\
\hline Un tipo de cáncer & 2 & $40,00 \%$ & 5 & $14,70 \%$ \\
\hline Dos o más tipos de cáncer & 0 & $0,00 \%$ & 2 & $5,90 \%$
\end{tabular}

$70 \%$

$9 \quad 16,40 \%$

$3 \quad 12,50 \%$

$30 \%$

\begin{tabular}{llrrrrrrrrrr}
\hline Dos o más tipos de cáncer & 0 & $0,00 \%$ & 2 & $5,90 \%$ & 6 & $10,90 \%$ & 2 & $8,30 \%$ & 3 & $33,30 \%$ \\
\hline Derecho & 2 & $40,00 \%$ & 11 & $32,40 \%$ & 20 & $36,40 \%$ & 10 & $41,70 \%$ & 4 & $44,40 \%$ \\
\hline Izquierdo & 2 & $40,00 \%$ & 8 & $23,50 \%$ & 14 & $25,50 \%$ & 4 & $16,70 \%$ & 2 & $22,20 \%$
\end{tabular}

\begin{tabular}{lllll}
\hline Istmo & 0 & $0,00 \%$ & 3 & $8,80 \%$ \\
\hline Derecho e izquierdo & 0 & $0,00 \%$ & 7 & $20,60 \%$
\end{tabular}

Porcentaje de

, $60 \%$

$\begin{array}{rrrr}14 & 25,50 \% & 4 & 16,70 \% \\ 2 & 3,60 \% & 0 & 0,00 \%\end{array}$

$\begin{array}{lllll}\text { Derecho e istmo } & 0 & 0,00 \% & 2 & 5,90 \%\end{array}$

$14 \quad 25,50 \%$

\begin{tabular}{lllll}
\hline Izquierdo e istmo & 0 & $0,00 \%$ & 2 & $5,90 \%$
\end{tabular}

$23,60 \%$

$7 \quad 29,20 \%$

o $0,00 \%$

Sinn

Derecho, izquierdo

$\begin{array}{lll}2,00 \% & 2,90 \%\end{array}$

$23,60 \%$

$14,20 \%$

Presencia de

Sin metástasis

metástasis

$$
\text { Gangliar }
$$

$\begin{array}{llll}3 & 60,00 \% & 21 & 61,80 \%\end{array}$

$1 \quad 1,80 \%$

$2 \quad 8,30 \% \quad 0 \quad 0,00 \%$

\begin{tabular}{llll}
\cline { 2 - 4 } & Ganglionar y tejidos & 0 & $0,00 \%$ \\
\hline \multirow{2}{*}{ Resultado de PAAF } & PAAF negativo & 1 & $25,00 \%$ \\
\cline { 2 - 4 } & PAAF positivo & 3 & $75,00 \%$ \\
\hline
\end{tabular}

Fuente: Base de datos Instituto Oncologico Dr. Juan Tanca Marengo - SOLCA 
TABLA2. PRUEBA DE CHI-CUADRADO DE PEARSON PARA LA DISTRIBUCIÓN POR GRUPOSDEEDAD.

\begin{tabular}{|c|c|c|}
\hline \multicolumn{3}{|c|}{ PRUEBAS DE CHI-CUADRADO DE PEARSON } \\
\hline \multirow{3}{*}{ Distribución por sexo } & Chi-cuadrado & 2,316 \\
\hline & $\mathrm{gl}$ & 4 \\
\hline & Sig. & $.678 a, b$ \\
\hline \multirow{3}{*}{ Distribución por zona } & Chi - cuadrado & 0,721 \\
\hline & $\mathrm{gl}$ & 4 \\
\hline & Sig. & $.949 \mathrm{a}$ \\
\hline \multirow{3}{*}{ Distribución por provincia } & Chi cuadrado & 36,597 \\
\hline & $\mathrm{gl}$ & 40 \\
\hline & Sig. & $.624 a, b$ \\
\hline \multirow{3}{*}{$\begin{array}{l}\text { Presencia de antecedentes } \\
\text { familiares de cáncer }\end{array}$} & Chi cuadrado & 17,106 \\
\hline & $\mathrm{gl}$ & 24 \\
\hline & Sig. & $.844 a, b$ \\
\hline \multirow{3}{*}{ Porcentaje de lóbulo afecto } & Chi cuadrado & 17,106 \\
\hline & $\mathrm{gl}$ & 24 \\
\hline & Sig. & $.844 a, b$ \\
\hline \multirow{3}{*}{ Presencia de metástasis } & Chi cuadrado & 4,969 \\
\hline & $\mathrm{gl}$ & 12 \\
\hline & Sig. & $.959 \mathrm{a}, \mathrm{b}$ \\
\hline \multirow{3}{*}{ Resultados de PAAF } & Chi cuadrado & 5,891 \\
\hline & $\mathrm{gl}$ & 4 \\
\hline & Sig. & $.207 a, b$ \\
\hline
\end{tabular}

*El estadístico de chi-cuadrado no es significativo.

Fuente: Base de datos Instituto Oncológico Juan Tanca Marengo - SOLCA

TABLA4. PRUEBA DEL CHI-CUADRADO PARA LA RELACIÓN ENTRE DISTRIBUCIÓN DE GRUPOS DE EDAD Y PRESENCIA DE METÁSTASIS.

\section{PRUEBAS DE CHI-CUADRADO}

\begin{tabular}{llll} 
& VALOR & GL & $\begin{array}{l}\text { SIG. ASINTÍtICA } \\
\text { BILATERAL }\end{array}$ \\
\hline Chi- cuadrado de Pearson & $4.969 \mathrm{a}$ & 12 & 0,959 \\
\hline Razón de verosimilitudes & 6,214 & 12 & 0,905 \\
\hline Asociación lineal por lineal & 0 & 1 & 0,993 \\
\hline N de casos válidos & 127 & &
\end{tabular}

Fuente: Base de datos Instituto Oncológico Juan Tanca Marengo - SOLCA.

*a 13 casillas (65.0\%) tienen una frecuencia esperada inferior a 5. Lafrecuencia mínima esperada es 24.

Se obtuvieron correlaciones entre variables cualitativas ordinales como metástasis y edad, en las cuales se midió si existía diferencia estadísticamente significativa mediante la prueba de Chi cuadrado, tabla3. Se consideró como estadísticamente significativo un valor $\mathrm{p}<0.05$. Tabla4 .

\section{RESULTADOS}

De la muestra obtenida (tabla 1), 105 pacientes correspondían al sexo femenino (82.7\%) y 22, al sexo masculino (17.3\%). La edad con mayor predominio de casos fue en un rango comprendido entre 37 - 52 años, siendo la edad media 46 años. La residencia de los habitantes no era exclusiva para la provincia del Guayas, esta provincia tiene la de mayor prevalencia de casos con 63 pacientes, se- guido de la provincia de Manabí con 30 pacientes. Un $60.66 \%$ de la población estudiada pertenecía a la zona urbana con 77 casos y un $39.37 \%$ a la zona rural con 50 casos.

Adicional a esto, 71 pacientes tenían ocupación doméstica ( $55,90 \%)$, le siguen profesionales que pertenecían a cargos ejecutivos 36 casos $(28,39 \%)$, estudiantes 15 casos $(11,81 \%)$, obreros 4 casos $(3,14 \%)$, profesional con riesgo laboral radiólogo 1 caso $(0.78 \%)$. Los datos de ocupación no se mencionan en la tabla antes mencionada. Entre los resultados de antecedentes familiares de cáncer se obtuvieron 93 pacientes que no referían enfermedad $(73,23 \%)$ y 34 pacientes positivos para cáncer en la familia, de los cuales 21 , solo referían 1 tipo con cáncer $(16,54 \%)$ y 13 con 2 o más variedades de cáncer $(10,24 \%)$.

A continuación se mencionan los tipos de cáncer hallados por el estudio: de mama, próstata, cérvix, gástrico, laringe, lengua, pulmón, tiroides, colon, vesícula biliar, páncreas, leucemia, linfoma Hodgkin, hepatocarcinoma, del sistema nervioso central.

En cuanto a la afectación lobular, hay una mayor frecuencia de afectación del lóbulo derecho en un $37 \%$ (47 casos), seguido de los lóbulos derecho e izquierdo en un $24,4 \%$ (31 casos), lóbulo izquierdo 23,6\% (30 casos), lóbulo izquierdo e istmo $4.7 \%$ (6 casos), lóbulo derecho e istmo 3.93\% (5 casos), istmo $3.93 \%$ ( 5 casos), y en un $2.36 \%$ ( 3 casos) toda la glándula.

Se estudió la presencia de metástasis en los pacientes de los cuales, $82(64,56 \%)$ no presentaron metástasis; $23,62 \%$ presentaron metástasis ganglionar; $7.08 \%$ con metástasis ganglionar y tejidos blandos; $4,72 \%$ metástasis de tejidos blandos. No se encontraron datos de metástasis a distancia. De los resultados de PAAF, 86 fueron positivos para malignidad $(67,71 \%$ ) y 22 PAAF se reportaron negativos (17,32\%). 19 pacientes no presentaron resultados de PAAF $(14,96 \%)$ en sus informes.

Se correlacionó la presencia de metástasis con los rangos de edad; se obtuvo la presencia de metástasis de tejidos blandos, ganglionar y combinada en mayor frecuencia en el rango de edad comprendida de 37 a 55 años, con un porcentaje de metástasis del $43,3 \%$; este valor se divide en $33.3 \%$, $40 \%, 44,4 \%$ para la metástasis de tejidos blando, ganglionar y combinada respectivamente. Es interesante recalcar que la metástasis de tejidos blandos se encuentra en iguales proporciones 
para los rangos de edad de (21-36 años), (37-52 años), (53-68 años) con un porcentaje de casos de $33.3 \%$. Los resultados de esta correlación se detallan en la tabla3.

\section{TABLA 3. CORRELACIÓN ENTRE DISTRIBUCIÓN POR GRUPOS DE EDAD Y PRESENCIA DE METÁSTASIS.}

\section{tABLA DE CONTINGENCIA DISTRIBUCIÓN POR GRUPOS dE EDAD * PRESENCIA} DE METíSTASIS

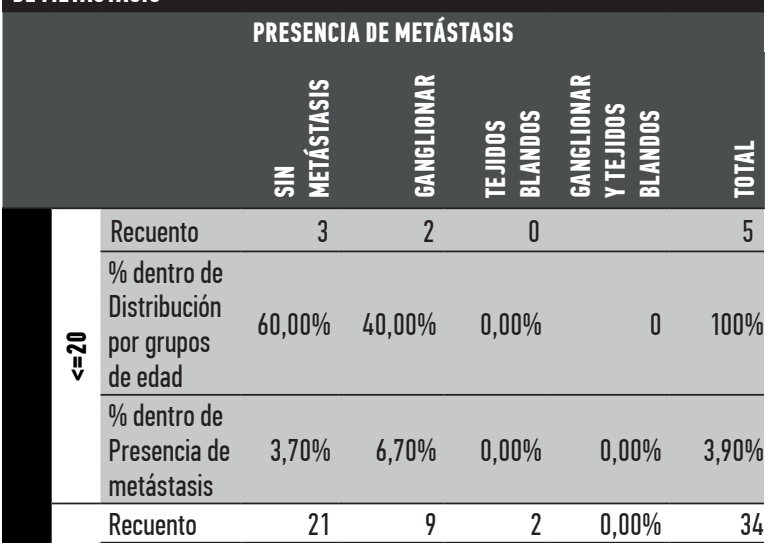

$\%$ dentro de

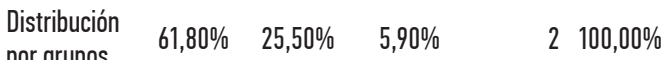

m por grupos

$\mp$ de edad

$\%$ dentro de $\quad-30,00 \%-33,30 \%-5,90 \%-26,80 \%$

Presencia de $\quad 25,60 \% \quad 30,00 \% \quad 33,30 \% \quad 5,90 \% \quad 26,80 \%$ metástasis

$\begin{array}{llllll}\text { Recuento } & 37 & 12 & 2 & 22,20 \% & 55\end{array}$

$\%$ dentro de

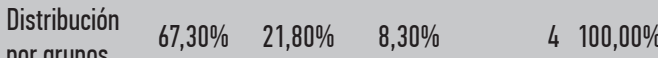

ก por grupos

de edad

$\%$ dentro de

Presencia de $\quad 45,10 \% \quad 40,00 \% \quad 3,60 \% \quad 7,30 \% \quad 43,40 \%$ metástasis

\begin{tabular}{llllll} 
Recuento & 14 & 5 & $33,30 \%$ & $44,40 \%$ & 24 \\
\hline
\end{tabular}

$\%$ dentro de

$\begin{array}{llll}\text { Distribución } & 58,30 \% & 22,20 \% & 3\end{array}$

o: por grupos

is de edad

$\%$ dentro de

Presencia de $\quad 17,10 \% \quad 16,70 \% \quad 8,30 \% \quad 12,50 \% \quad 18,90 \%$ metástasis

\begin{tabular}{llllll} 
Recuento & 7 & 2 & $33,30 \%$ & $33,30 \%$ & 9 \\
\hline
\end{tabular}

\begin{tabular}{llllll} 
Recuento & 7 & 2 & $33,30 \%$ & $33,30 \%$ & 9 \\
\hline$\%$ dentro de & & & &
\end{tabular}

$\begin{array}{lllll}\text { Distribución } & 77,80 \% & 22,20 \% & 0 & 0\end{array}$

$\pm$

por grupos

$\%$ dentro de

$\begin{array}{lllll}\text { Presencia de } \quad 8,50 \% & 6,70 \% & 0,00 \% & 0,00 \% & 7,10 \%\end{array}$ metástasis

\begin{tabular}{|c|c|c|c|c|c|}
\hline Recuento & 82 & 30 & $0,00 \%$ & $0,00 \%$ & 127 \\
\hline $\begin{array}{l}\text { \% dentro de } \\
\text { Distribución } \\
\text { por grupos } \\
\text { de edad }\end{array}$ & $64,60 \%$ & $23,60 \%$ & $4,70 \%$ & 9 & $100,00 \%$ \\
\hline $\begin{array}{l}\% \text { dentro de } \\
\text { Presencia de } \\
\text { metástasis }\end{array}$ & $100,00 \%$ & $100,00 \%$ & $100,00 \%$ & $100,00 \%$ & $100,00 \%$ \\
\hline
\end{tabular}

Fuente: Base de datos Instituto Oncológico Dr. Juan Tanca Marengo - SOLCA
DISCUSIÓN

El estudio reporta principalmente datos de la región costa teniendo una incidencia estimada en 1,64 x 100.000 habitantes dentro de esta región; la provincia con mayor incidencia de cáncer en el 2014 fue Manabí con aproximadamente 2,19 x100.000 habitantes, seguida por la provincia del Guayas con una incidencia de 1,72 x 100000 habitantes ${ }^{20}$. La incidencia del cáncer papilar es mucho más elevada en mujeres que en varones, lo que concuerda con estadísticas a nivel mundial. La edad de presentación de esta patología es la misma en ambos sexos, con mayor predominio de casos en un rango entre 37 - 52 años, siendo la edad media 46 años. Este análisis se corresponde con los resultados del estudio SEER de Estados Unidos de Norteamérica, con datos del 2012 en el cual el rango de edad fue de 45 a 54 años siendo la edad media 50 años ${ }^{17}$.

El hecho de que la incidencia del cáncer de tiroides sea de 2 a 3 veces mayor en mujeres que en varones, permite inferir que las hormonas femeninas tienen un papel importante para producir esta patología.

Diferentes estudios han observado la relación urbano - rural y afectación de este cáncer; los resultados del presente estudio arroja que un $60.66 \%$ de la población afectada pertenecía a la zona urbana lo que denota una fuerte inclinación hacia este sector lo que permite inferir que el tipo de vida y contaminación de estas áreas tengan influencia en la génesis de este cáncer. Los factores predisponentes para esta situación no se mencionan en la literatura. A nivel de Europa no se han encontrado diferencias significativas a nivel de las zonas urbanas y rurales. ${ }^{1,2,4}$.

No existe información concluyente con respecto al riesgo ocupacional y cáncer de tiroides en la bibliografía utilizada. La relación entre los pacientes con cáncer papilar de tiroides y su profesión está en discusión ya que solo 1 paciente tenía una ocupación de alto riesgo para una patología como el cáncer, por su exposición diaria a radiación ${ }^{2,4,21}$.

De los 127 pacientes analizados, se realizaron 119 PAAF $(85,04 \%)$ de los cuales, 86 fueron positivos para malignidad $(67,71 \%)$ y 22 PAAF se reportaron negativos $(17,32 \%) .19$ pacientes no presentaban resultados de PAAF $(14,96 \%)$ en sus informes. Otro hallazgo importante es que sólo $38 \%$ de los PAAF fueron realizados dentro de SOLCA Guayaquil; el 
$62 \%$ fueron realizados en los hospitales que derivaron los pacientes a este instituto. Al comparar los PAAF realizados en SOLCA, Guayaquil y los derivados, se pudo observaruna mayor sensibilidad en los que fueron derivados del $80 \%$, y los de SOLCA fue de $79 \%$, lo cual no es una diferencia significativa entre estos centros. Esto podría sugerir que la cobertura que SOLCA y los centros de derivación, brindan un buen pesquisaje de citología positiva para malignidad. Pero hay que aclarar que estos datos de PAAF lastimosamente no son totalmente fiables ya que no se cuenta con la totalidad de los estudios de estas instituciones, pero cumplen como una buena aproximación para este estudio citológico.

Un mayor porcentaje de la muestra no presentó metástasis al estudio anatomopatológico, correspondiendo al $64,56 \%$. Un $23,62 \%$ presentó metástasis ganglionar, dato que se correlaciona con el estudio INCATIR, chile, en donde sólo el 29,1\% de los cánceres papilares tuvieron metátesis a nivel ganglionar. El lóbulo derecho tuvo mayor afectación en un $37 \%$ de los casos, seguido por el izquierdo con el 23,6\%, y casos multicéntricos fueron los más frecuentes con un 41,39\%, el istmo representó solo el $3.93 \%$ de los casos ${ }^{1}$. Un alto porcentaje de los casos no reportó antecedentes familiares de cáncer y de los pocos que sí lo hicieron, los más frecuentes fueron cáncer de mama y de tiroides. Por lo tanto, tener antecedentes familiares de cáncer tiroides aumentaría el riesgo de padecerlo, pero no se conocen las bases genéticas de este factor ${ }^{16}$.

De acuerdo a la correlación entre presencia de metástasis y edad (tabla 3, figura 1), se obtuvo que la presencia de metástasis es más frecuente en edades comprendidas entre 37 a 55 años. De los pacientes $>70$ años (9 casos), 7 de ellos no presentaron metástasis y 2 si lo hicieron, siendo de afectación ganglionar, diferenciándose así de los demás grupos etarios que tenían porcentajes más altos de metástasis ${ }^{2}$.

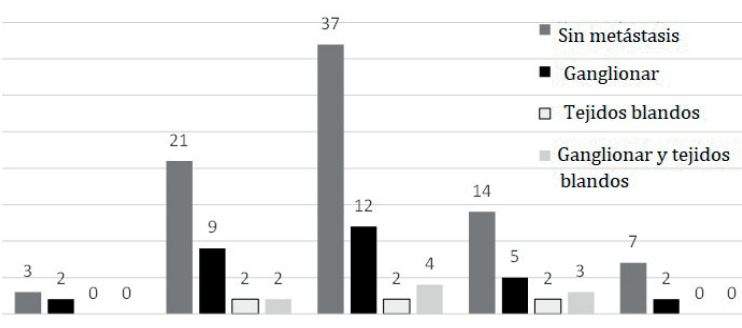

Figura 1. Correlación entre grupos de edad y la presencia de metástasis. Los grupos etarios de 21-36 años y 37- 52 años son los que tienen más predisposición a presentar metástasis ganglionar. Y la presencia de metástasis ganglionar se encuentra mayormente en el grupo de 37- 52 años. Fuente: Base de datos Instituto Oncológico Dr. Juan Tanca Marengo - SOLCA.
Una de las principales limitaciones de este estudio es la revisión de carácter retrospectivo basada en las historias clínicas y registros del hospital. Las variables analizadas son de carácter objetivo y sólo son reconocidas como válidas cuando se recogieron en documentos totalmente íntegros. Respecto al papel pronóstico de muchas de ellas, sólo se evaluó su asociación estadística bivariante y se realizaron pocos análisis multivariantes debido a la inestabilidad de los modelos de regresión y bajo número de pacientes.

\section{CONCLUSIÓN}

De acuerdo a los datos analizados en el presente estudio se concluye que el carcinoma papilar de tiroides en población ecuatoriana se presenta con mayor frecuencia en mujeres, con una edad media de 46 años, con residencia urbana como factor influyente en la patología, con afectación tiroidea unilobular de predominio derecho, con escasa presencia de metástasis. No hay relación de los antecedentes de cáncer en la familia u ocupación, con la aparición de esta neoplasia.

\section{RECOMENDACIONES}

Es necesario un estudio con un mayor tamaño de muestra, que abarque varios centros oncológicos del Ecuador para que los resultados sean más representativos de nuestro país. La recolección de datos al momento del ingreso de un paciente al sistema hospitalario debe ser más minuciosa en cuanto a los antecedentes patológicos familiares y personales, mayor especificación en las actividades que realiza el paciente y posibles factores de riesgo agregados.

\section{REFERENCIAS BIBLIOGRÁFICAS}

1. Jorge SapunarZ.1,2, Sergio Muñoz N.2,3, Juan Carlos Roa S.4. Epidemiología del cáncer de tiroides enChile. Resultados del estudio INCATIR*. RevMed Chile 2014; 142: 1099-1105.

2. Dan L. Longo, Dennis L. Kasper, J. Larry Jameson, Anthony S. Fauci, Stephen L. Hauser, Joseph Loscalzo, Harrison. Principios de Medicina Interna, 18ed, 2012

3. Granados García, León Takahashi, Guerrero Huerta y Taissoun Aslan. Servicio de Cabeza y Cuello, Instituto Nacional de Cancerología, México, D.F. Cáncer diferenciado de tiroides: una antigua enfermedad con nuevos conocimientos. Gaceta Médica de México. 2014;150:65-77.

4. V. Lope Carvajal y M. Pollán Santamaría. Área de Epidemiología Ambiental y Cáncer. Centro Nacional de Epidemiología del cáncer diferenciado de tiroides. Instituto de Salud Carlos III. Madrid. España. EndocrinolNutr. 2005;52(Supl 1):2-10 
5. Vargas Uricoechea, Herrera Chaparro, Meza Cabrera, Agred; Epidemiología del cáncer de tiroides. Analisis de resultados en Sudamérica y Colombia. Revista Medicina; Vol 37 nº 2, año 2015.

6. Liberman G. Unidad de Endocrinología, Departamento de Medicina Interna. Clínica Las Condes. Prevalencia e incidencia de los principales transtornos endocrinos y metabólicos. Revista Médica Clínica Las Condes / vol. 24 nํ5 / Septiembre 2013

7. Horn-Ross PL, Chang ET, Clarke CA, Keegan TH, Rull RP, Quach T, Gomez SL. Nativity and papillary thyroid cancer incidence rates among Hispanic women in California. Cancer 2012; 118(1): 216-222.

8. Pellegriti G, Frasca F, Regalbuto C, Squatrito S, Vigneri R. Worldwide increasing incidence of thyroid cancer: update on epidemiology and risk factors. J Cancer Epidemiol.2013;2013:965212. doi: 1155/2013/965212.

9. Arrieta Vasquez. Incidencia y prevalencia del cáncer papilar y folicular de tiroides del hospital universitario Dr.Luisrazzetti. Universidad de oriente núcleo de anzoátegui escuela de medicina departamento de cirugía, Barcelona, noviembre de 2010.

10. Brito AS, Coeli CM, Barbosa FS, Caetano R, Santos MO, Vaisman M. Estimates of thyroid cancer incidence in Brazil: an approach using polynominal models. Cad Saúde Pública, Río de Janeiro. 2011: 27(7): 1441- 1444.

11. Chala1, Franco2, Aguilar3, Cardona4. Estudio descriptivo de doce años de cáncer de tiroides, Manizales, Colombia. RevColomb Cir. 2010;25:276-89

12. Faure, Soutelo, Faraj, Lutfi, Juvenal. Servicio Endocrinología, Hospital Churruca-Visca y Comisión Nacional de Energía Atómica, Buenos Aires, Argentina. Estimación de la Incidencia de Cáncer de Tiroides en Capital Federal y el Gran Buenos Aires (período 2003-2011).Revista Argentina de Endocrinología y Metabolismo 2012 por la Sociedad Argentina de Endocrinología y Metabolismo.
13. Iglesias Díaz, Lau López, García García .Incidencia del cáncer de tiroides en la consulta central de tiroides, últimos nueve años en Pinar del Río. Rev. Ciencias Médicas. Abril 2008; 12(1)

14. Fernández Cantón, Sánchez Díaz, González Flores, Cruz Cruz, Cortés Ramírez. Perfil epidemiológico de los tumores malignos en México. Secretaría de salud subsecretaría de prevención y promoción de la salud. Dirección general de epidemiología. Junio, 2011

15. Hurtado-López, Basurto-Kuba, Montes de OcaDurán, Pulido-Cejudo, Vázquez-Ortega, AthiéGutiérrez. Prevalencia de nódulo tiroideo en el valle de México. Hospital General de México, Secretaría de Salud, México, D. F, CirCir 2011;79:114-117.

16. American Cancer society, Guía del cáncer tiroide, 2014. http://www.cancer.org/

17. The Surveillance, Epidemiology, and End Results (SEER) Program of the national Cancer Institute (NCI) http://seer.cancer.gov

18. Sociedad de lucha contra el cáncer/ Registro Nacional de Tumores. Cueva Ayala P, Yépez Maldonado J. Editores 2014. 40. Epidemiología del cáncer en Quito 2006-2010. Quito. 15 ed.

19. Instituto Nacional del Cáncer (NIH) de los Institutos Nacionales de Salud de los E.E.U.U. Estatificación del cáncer. Revisión 6 de enero de 2015. http://www. cancer.gov

20. Instituto Nacional de Estadísticas y Censos. http:// www.ecuadorencifras.gob.ec

21. Guyton, A.C. Hall, J.E. Tratado de fisiología médica. 12를. Madrid: Elsevier; 2011 